\title{
Changing Global Trends in Seizure Outcomes Following Resective Surgery for Tuberous Sclerosis in Children with Medically Intractable Epilepsy
}

\author{
George M. Ibrahim, , 2, 3 Aria Fallah,, 3,4 0 . Carter Snead, ${ }^{\text {,, 3,5 }}$ and James T. Rutka ${ }^{1,3}$ \\ ${ }^{1}$ Division of Neurosurgery, Department of Surgery, University of Toronto, Toronto, ON, Canada \\ ${ }^{2}$ Institute of Medical Sciences, University of Toronto, Toronto, ON, Canada \\ ${ }^{3}$ Hospital for Sick Children Research Institute, Suite 1503, 555 University Avenue, Toronto, ON, Canada M5G 1X8 \\ ${ }^{4}$ Department of Clinical Epidemiology and Biostatistics, McMaster University, Hamilton, ON, Canada \\ ${ }^{5}$ Department of Neurology, Hospital for Sick Children, Toronto, ON, Canada
}

Correspondence should be addressed to James T. Rutka, james.rutka@sickkids.ca

Received 9 September 2012; Accepted 30 October 2012

Academic Editor: Raidah S. Albaradie

Copyright ( 2012 George M. Ibrahim et al. This is an open access article distributed under the Creative Commons Attribution License, which permits unrestricted use, distribution, and reproduction in any medium, provided the original work is properly cited.

\begin{abstract}
Introduction. Tuberous sclerosis (TS) is the leading cause of genetic epilepsy worldwide. Here, we evaluate changes in seizure outcomes following resective epilepsy surgery in children with TS over time. Methods. A systematic review of the literature was performed to identify studies reporting seizure outcomes following resective epilepsy surgery in children with TS. Using an individual participant meta-analysis approach, seizure outcomes and associated covariates were combined. Multivariate logistic regression was used to determine significant associations between seizure outcomes and time of surgery. Results. Twenty studies from 1966 to present, yielding 186 participants, met the inclusion criteria for the study. On univariate analysis, there was a significant improvement in seizure outcomes in children who underwent resective epilepsy surgery within the last 15 years compared to older cohorts (chi-square 4.1; $P=0.043$ ). On multivariate analysis, adjusting for length of followup, this trend was not significant (OR $0.52 ; 95 \%$ CI $0.23-1.17 ; P=0.11$ ). In the last 15 years, a greater proportion of younger children aslo underwent resective surgery compared to older cohorts (OR 0.93; 95\% CI 0.89-0.97; $P<0.01$ ). Conclusions. A trend towards improved seizure outcomes following resective surgery for TS was observed from 1966 to present on multivariate analysis.
\end{abstract}

\section{Introduction}

Tuberous sclerosis complex (TS) is the leading cause of genetic childhood epilepsy worldwide [1]. The condition is characterized by inactivating mutations in TSC1, encoding hamartin, or TSC2, encoding tuberin [1]. These lead to downstream hyperactivation of the mammalian target of rapamycin (mTOR) pathway and associated kinase signalling cascades, resulting in abnormal cortical development and epilepsy in the majority of affected children. The neurological manifestations of TS are associated with considerable morbidity [2]. Specifically, $50-80 \%$ of children with epilepsy secondary to TS may be refractory to medical treatment [3, 4]. Furthermore, seizure frequency and epilepsy severity have been previously related to the extent of cognitive impairment in children with TS [5].
Resective surgical strategies are increasingly utilized in children with TS due to the medical and psychosocial burden of medically intractable epilepsy. Surgical treatment has been shown to result in a significant control of epilepsy and improvements in the quality of life of children with TS [6]. These surgical strategies have, however, changed dramatically over the last several decades. This is largely due to advances in localization strategies, which are particularly important in TS, as multiple tubers are often present and differentiating the epileptogenic tuber from others can be challenging. Standard localization modalities for the identification of epileptogenic cortex in TS may include magnetic resonance imaging (MRI), scalp and intracranial electroencephalography (EEG), positron emission tomography (PET), magnetoencephalography (MEG), and single-photon emission computed tomography (SPECT). 
The last 15 years (1996-2011) specifically have also seen numerous advancements in noninvasive and invasive methods for the localization of eloquent cortex, allowing for more effective and safer surgical treatments of epilepsy. These include advances in the aforementioned technologies and the addition of functional MRI, diffusion tensor imaging-fiber tractography, and diffusion-weighted imaging. During this time period, there have also been considerable refinement of techniques and a reliance on the concordance of multiple localization modalities to guide surgical strategies. It is unclear, however, whether advances in technology have had a significant impact on seizure outcomes in children with TS. The current paper utilizes independent participant data meta-analysis methodology to determine whether seizure outcomes have improved over the last 50 years following resective surgery for children with TS.

\section{Methods}

A systematic review of literature was performed to identify studies reporting seizure outcomes in children with medically intractable epilepsy over the last 50 years. The following electronic databases were searched for relevant articles with no date or language restrictions: MEDLINE, EMBASE, CINAHL, and Web of Science. The search was performed in October 2011. The search strategy was limited to humans and incorporated the following terms: "tuberous sclerosis," "epilepsy surgery," "seizure outcomes," "Engel classification," and "predictors." The inclusion criteria for studies were (1) case-controlled or cohort studies; (2) consecutive participants; (3) at least $90 \%$ of participants be under the age of 19 at time of surgery; (4) at least $90 \%$ of subjects have TS; (5) at least $90 \%$ of subjects have undergone resective epilepsy surgery; and (6) seizure outcomes are reported. We chose high thresholds (i.e., 90\%) for inclusion in the metaanalysis to ensure that the data was not biased by children with other pathologies or those who did not undergo a resective surgical procedure. Additionally, case reports, reviews, mixed studies (adults and children), and studies including children who have undergone previous resective or palliative (i.e., corpus callosotomy, multiple subpial transection, vagal nerve stimulator insertion) surgery were excluded. Title and abstract screening as well as full text review were performed independently and in duplicate. Disagreements between reviewers were resolved by joint review and consensus with respect to the eligibility criteria.

An independent participant data meta-analysis (IPDMA) approach was utilized whereby the raw participant-level data were obtained from each study and synthesized. The primary outcome of interest was whether seizure outcomes improved in published studies over time. Specifically, individual participants were dichotomized based on their date of surgery (defined for each participant by subtracting their individual length of followup from the year of article participation) into recent cohorts (surgery within the last 15 years) and older cohorts (surgery greater than 15 years ago). Differences in seizure outcome were compared between the two cohorts. Seizure outcome was defined as satisfactory (Engel I-II) and unsatisfactory (Engel III-IV) at the last followup.
Data are presented as frequencies or means with standard deviations. The chi-squared test was employed for univariate analysis to determine differences in seizure outcomes between recent and older cohorts. Multivariate logistic regression was used to adjust for the length of followup, as those with longer follow-up times may be more likely to have recurrence of seizure and therefore a worse Engel score. A $P$ value less than 0.05 was accepted as statistically significant. All data were analyzed using SAS statistical software 9.3 (Cary, NC, USA).

\section{Results}

We identified 9863 citations from the electronic database queries, of which 241 full text articles were reviewed. Twenty articles reporting on 181 participants were ultimately included in the study (Table 1). The subjects' demographics are presented in Table 2 . The mean age of children was $9.8 \pm$ 8.8 years. Thirteen articles $(65 \%)$ originated from North America, while five (25\%) and three (15\%) originated from Europe and Asia, respectively. One hundred and twenty-six $(70 \%)$ of these subjects had a satisfactory seizure outcome with a mean duration of followup of 2.3 years. The majority of studies performed a surgical strategy involving tuberectomy or lobectomy (70\%); however, several studies also included multilobar resections (50\%) and hemispherectomy $(10 \%)$.

Using an IPDMA approach, the surgical outcomes of participants were concatenated and dichotomized as favourable (Engel I-II) and unfavourable (Engel III-IV) and compared in recent and older cohorts. On univariate analysis, recent cohorts of children undergoing resective procedures (within the last 15 years) were significantly more likely to achieve a favourable outcome compared to older cohorts (older cohorts to 15 years ago; chi-squared $=4.1 ; P=0.04$; Figure 1). Multivariate logistic regression was applied to determine differences in seizure outcomes between the two time cohorts, while adjusting for each participant's duration of following. There was a trend towards better seizure outcomes in recent cohorts following adjusting for duration of followup, which did not reach the threshold for statistical significance (odds ratio (OR) $0.52 ; 95 \%$ confidence interval (CI) $0.23-1.17 ; P=0.11$.

When the epoch of surgery was entered into the logistic regression model as the dependent variable, it was also determined that the age of children at the time of surgery was significantly different between the two epochs. Over the last 15 years, a greater proportion of younger children underwent a resective surgery compared to older cohorts (OR 0.93; 95\% CI $0.89-0.97 ; P<0.01)$. There were no significant differences between outcomes in North America and elsewhere $(P=$ 0.45).

\section{Discussion}

This exploratory analysis determined that there was a nonsignificant trend on multivariate analysis towards improved seizure outcomes over the last 15 years in children with medically intractable epilepsy secondary to TS following 
TABLE 1: Included studies of outcomes following resective surgery in children with tuberous sclerosis and intractable epilepsy.

\begin{tabular}{|c|c|c|c|c|}
\hline $\begin{array}{l}\text { First author } \\
\text { (reference) }\end{array}$ & Year of publication & Country of origin & Number of subjects & Percentage seizure-free \\
\hline Perot [21] & 1966 & Canada & 7 & 43 \\
\hline Bebin [22] & 1993 & USA & 7 & 71 \\
\hline Avellino [23] & 1997 & USA & 9 & 50 \\
\hline Baumgartner [24] & 1997 & USA & 4 & 25 \\
\hline Guerreiro [25] & 1998 & Canada & 12 & 67 \\
\hline Asano [26] & 2000 & USA & 7 & 71 \\
\hline Koh [27] & 2000 & USA & 13 & 73 \\
\hline Karenfort [5] & 2002 & Germany & 8 & 88 \\
\hline Kagawa [28] & 2005 & USA & 17 & 71 \\
\hline Lachhwani [29] & 2005 & USA & 17 & 71 \\
\hline Jansen [3] & 2006 & Netherlands & 3 & 67 \\
\hline Kamimura [30] & 2006 & Japan & 3 & 100 \\
\hline Weiner [31] & 2006 & USA & 25 & 92 \\
\hline Jansen [32] & 2007 & Netherlands & 6 & 67 \\
\hline Teutonico [33] & 2008 & Italy/USA & 11 & 45 \\
\hline Major [34] & 2009 & USA & 3 & 67 \\
\hline Wen [35] & 2009 & China & 4 & 75 \\
\hline van der Heide [36] & 2010 & Netherlands & 6 & 67 \\
\hline Liang [37] & 2010 & China & 17 & 76 \\
\hline Aboian [4] & 2011 & USA & 6 & 50 \\
\hline
\end{tabular}

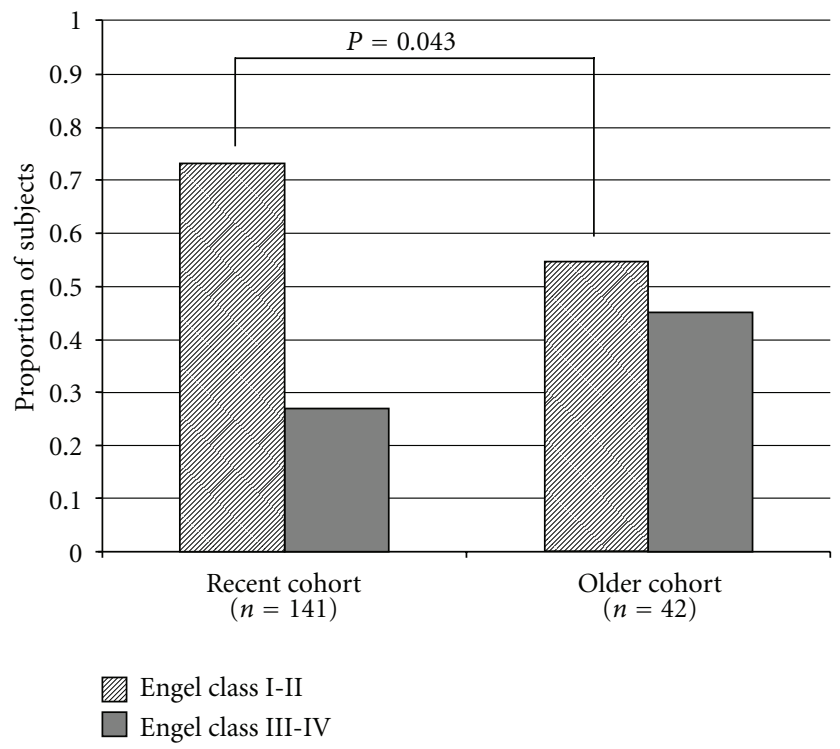

Figure 1: Proportion of children with tuberous sclerosis achieving favourable seizure outcome (Engel I-II) following resection surgery in recent and older cohorts. Recent cohorts demonstrate significantly improved seizure outcomes on univariate analysis.

resective surgery. Furthermore, we find that compared to older cohorts, more recent cohorts included children with a younger mean age. The current analysis is the largest study evaluating trends in seizure outcome in TS, concatenating data from seven countries and three continents using an
TABle 2: Pooled patient demographics.

\begin{tabular}{lc}
\hline Variable & Frequency/mean \\
\hline Gender & \\
Male & $52 \%$ \\
Female & $48 \%$ \\
Age at surgery & $9.8 \pm 8.8$ \\
Surgical strategy* & \\
Tuberectomy/lobectomy & $70 \%$ \\
Multilobar resection & $50 \%$ \\
Hemispherectomy & $10 \%$ \\
\hline
\end{tabular}

* Percentages expressed as a proportion of studies employing the surgical strategies.

IPDMA approach. These findings should inform the academic community regarding advances in the surgical treatment of children with TS over the last 50 years.

The last 15 years have seen numerous advances in diagnostic imaging and localization modalities. For instance, it is well described that tubers may be conspicuous on T2weighted and FLAIR MRI sequences; however, it has been recently reported that epileptic tubers demonstrate increased apparent diffusion coefficients and tensor properties [7, 8]. Additionally, the evolution of MEG techniques, especially the coregistration of MEG dipoles with MRI, has resulted in improved seizure outcomes in individual studies [3]. Furthermore, novel markers have been developed to identify the epileptic tuber, including alpha-[11C]-methyl-ltryptophan (AMT) PET, on the basis of findings of increased 
concentrations of serotonin and serotonin-immunoreactive processes in epileptic cortex [9]. Finally, there has also been a concerted effort to amalgamate numerous localization modalities to the localization of the epileptogenic tuber. For example, PET/MRI coregistration has been reported by some groups to result in improved seizure outcomes [10]. Increasingly, centres are relying upon the concordance of numerous modalities in the surgical decision-making processes.

While there was a significant difference in seizure outcomes between recent and older surgical cohorts on univariate analysis, this trend did not reach statistical significance on multivariate modelling. There may be several explanations for this finding. Firstly, although advancements in technologies and surgical techniques have been achieved over the last 15 years, there remains a significant heterogeneity in their application to the presurgical evaluation of children with epilepsy $[11,12]$. Our results represent a global average based on the concatenation of results from 20 published series. The finding of a trend towards improved seizure outcomes is therefore in and of itself highly encouraging. Secondly, our dichotomization cutoff of 15 years was largely based on the timing of the widespread introduction of novel localization technologies to presurgical evaluation of medically intractable epilepsy. It is possible that a time lag in the application of these innovations or a "learningcurve" at centres that have adapted these technologies may delay improvements in surgical outcomes [13, 14]. Thirdly, the widespread adoption of invasive monitoring by comprehensive epilepsy centres has permitted the treatment of more complex patients that would have previously been excluded from standard treatments such as lesionectomies (tubectomies) [15]. It is possible that the lack of a significant improvement is a reflection of the increasing complexity of surgically treated TS made possible by advancements in invasive monitoring technology. Finally, TS is a challenging condition, whereby nonresected tubers may become independently epileptogenic. The finding that seizure outcomes between recent and older cohorts were not significant upon statistical adjustment for length of followup may reflect the phenomenon that children with TS often develop secondary epilepsy syndromes on prolonged followup.

In the contemporary practice of pediatric epilepsy surgery, there has also been a greater appreciation of the detrimental effects of seizures on childhood development $[16,17]$. Furthermore, a longer duration of epilepsy is associated with a lesser likelihood of seizure freedom; therefore, early surgical intervention in young patient cohorts has been advocated by numerous groups [18-20]. Indeed, it has been shown that surgical treatment improves the quality of life of children with TS and that, given the choice, the vast majority (94\%) of parents would choose to undergo the resective procedure once again [6]. The current finding of significantly younger children undergoing resective procedures in the more recent cohort suggests that there have been improvements in the triage, referral, and presurgical workup of younger children with intractable epilepsy. This of course may also contribute to the trend in improvements in seizure outcomes observed.

The most important limitation of the current article is the possibility that appropriate manuscripts were missed in the systematic review search strategy. Secondly, because we rely on the variables collected by the authors of the original articles, we were unable to include additional potential covariates in the multivariate regression model. Furthermore, there were considerably fewer children in the older cohort compared to the more recent cohort. Finally, as these data are based on retrospective series, several biases inherent to such methodologies may also limit the findings presented herein. A significant strength of the current methodology is the use of the IPDMA approach to concatenate individual participant data from the selected articles, thereby bypassing numerous publication biases.

\section{Conclusions}

Compared to older cohorts, children that have undergone resective surgery for medically intractable epilepsy secondary to tuberous sclerosis in the past 15 years demonstrate improved seizure outcomes on univariate, but not multivariate analysis. A greater proportion of younger children was also included in the recent cohort. This study hopes to inform the academic community regarding advancements in the surgical treatment of TS over time. Further research and policy are necessary to improve seizure outcomes and subsequent analyses should reexamine the effects of such advancements on future patient populations.

\section{Acknowledgments}

The authors would like to gratefully acknowledge the help of Alireza Mansouri, Shanil Ebrahim, and Deven Reddy who contributed to abstract screening.

\section{References}

[1] P. Curatolo, R. Bombardieri, and S. Jozwiak, "Tuberous sclerosis," The Lancet, vol. 372, no. 9639, pp. 657-668, 2008.

[2] L. Hallett, T. Foster, Z. Liu, M. Blieden, and J. Valentim, "Burden of disease and unmet needs in tuberous sclerosis complex with neurological manifestations: systematic review," Current Medical Research and Opinion, vol. 27, no. 8, pp. 15711583, 2011.

[3] F. E. Jansen, G. Huiskamp, A. C. van Huffelen et al., "Identification of the epileptogenic tuber in patients with tuberous sclerosis: a comparison of high-resolution EEG and MEG," Epilepsia, vol. 47, no. 1, pp. 108-114, 2006.

[4] M. S. Aboian, L. C. Wong-Kisiel, M. Rank, N. M. Wetjen, E. C. Wirrell, and R. J. Witte, "SISCOM in children with tuberous sclerosis complex-related epilepsy," Pediatric Neurology, vol. 45, no. 2, pp. 83-88, 2011.

[5] M. Karenfort, B. Kruse, H. Freitag, H. Pannek, and I. Tuxhorn, "Epilepsy surgery outcome in children with focal epilepsy due to tuberous sclerosis complex," Neuropediatrics, vol. 33, no. 5, pp. 255-261, 2002.

[6] J. Roth, A. Olasunkanmi, W. S. Macallister et al., "Quality of life following epilepsy surgery for children with tuberous sclerosis complex," Epilepsy and Behavior, vol. 20, no. 3, pp. 561-565, 2011.

[7] F. E. Jansen, K. P. J. Braun, O. van Nieuwenhuizen et al., "Diffusion-weighted magnetic resonance imaging and identification of the epileptogenic tuber in patients with tuberous 
sclerosis," Archives of Neurology, vol. 60, no. 11, pp. 1580-1584, 2003.

[8] P. S. Chandra, N. Salamon, J. Huang et al., "FDG-PET/MRI coregistration and diffusion-tensor imaging distinguish epileptogenic tubers and cortex in patients with tuberous sclerosis complex: a preliminary report," Epilepsia, vol. 47, no. 9, pp. 1543-1549, 2006.

[9] D. C. Chugani, H. T. Chugani, O. Muzik et al., "Imaging epileptogenic tubers in children with tuberous sclerosis complex using $\alpha$-[11C]methyl-L-tryptophan positron emission tomography," Annals of Neurology, vol. 44, no. 6, pp. 858-866, 1998.

[10] J. Y. Wu, N. Salamon, H. E. Kirsch et al., "Noninvasive testing, early surgery, and seizure freedom in tuberous sclerosis complex," Neurology, vol. 74, no. 5, pp. 392-398, 2010.

[11] A. S. Harvey, J. H. Cross, S. Shinnar, G. W. Mathern, and ILAE Pediatric Epilepsy Surgery Survey Taskforce, "Defining the spectrum of international practice in pediatric epilepsy surgery patients," Epilepsia, vol. 49, no. 1, pp. 146-155, 2008.

[12] G. M. Ibrahim, B. W. Barry, A. Fallah et al., "Inequities in access to pediatric epilepsy surgery: a bioethical framework," Neurosurgical Focus, vol. 32, no. 3, article E2, 2012.

[13] L. Morgenstern, "Warning! Dangerous curve ahead: the learning curve," Surgical Innovation, vol. 12, no. 1, pp. 101-103, 2005.

[14] G. M. Ibrahim, A. Fallah, O. C. Snead 3rd, J. Drake, J. T. Rutka, and M. Bernstein, "The use of high frequency oscillations to guide neocortical resections in children with medically-intractable epilepsy: how do we ethically apply surgical innovations to patient care?" Seizure, vol. 21, no. 10, pp. 743-747, 2012.

[15] S. Goldring, "A method for surgical management of focal epilepsy, especially as it relates to children," Journal of Neurosurgery, vol. 49, no. 3, pp. 344-356, 1978.

[16] G. L. Holmes and Y. Ben-Ari, "Seizures in the developing brain: perhaps not so benign after all," Neuron, vol. 21, no. 6, pp. 1231-1234, 1998.

[17] K. J. Meador, "Cognitive outcomes and predictive factors in epilepsy," Neurology, vol. 58, no. 8, supplement 5, pp. S21-S26, 2002.

[18] G. M. Ibrahim, A. Fallah, O. C. Snead 3rd et al., "Ethical issues in surgical decision making concerning children with medically intractable epilepsy," Epilepsy and Behavior, vol. 22, no. 2, pp. 154-157, 2011.

[19] C. Helmstaedter and C. E. Elger, "Chronic temporal lobe epilepsy: a neurodevelopmental or progressively dementing disease?" Brain, vol. 132, no. 10, pp. 2822-2830, 2009.

[20] R. Jonas, S. Nguyen, B. Hu et al., "Cerebral hemispherectomy: hospital course, seizure, developmental, language, and motor outcomes," Neurology, vol. 62, no. 10, pp. 1712-1721, 2004.

[21] P. Perot, B. Weir, and T. Rasmussen, "Tuberous sclerosis: surgical therapy for seizures," Archives of Neurology, vol. 15, no. 5, pp. 498-506, 1966.

[22] E. M. Bebin, P. J. Kelly, M. R. Gomez, P. Genton, and C. K. Benninger, "Surgical treatment for epilepsy in cerebral tuberous sclerosis," Epilepsia, vol. 34, no. 4, pp. 651-657, 1993.

[23] A. M. Avellino, M. S. Berger, R. C. Rostomily, C. M. Shaw, and G. A. Ojemann, "Surgical management and seizure outcome in patients with tuberous sclerosis," Journal of Neurosurgery, vol. 87, no. 3, pp. 391-396, 1997.

[24] J. E. Baumgartner, J. W. Wheless, S. Kulkarni et al., "On the surgical treatment of refractory epilepsy in tuberous sclerosis complex," Pediatric Neurosurgery, vol. 27, no. 6, pp. 311-318, 1997.

[25] M. M. Guerreiro, F. Andermann, E. Andermann et al., "Surgical treatment of epilepsy in tuberous sclerosis: strategies and results in 18 patients," Neurology, vol. 51, no. 5, pp. 12631269, 1998.

[26] E. Asano, D. C. Chugani, O. Muzik et al., "Multimodality imaging for improved detection of epileptogenic foci in tuberous sclerosis complex," Neurology, vol. 54, no. 10, pp. 1976-1984, 2000.

[27] S. Koh, P. Jayakar, C. Dunoyer et al., "Epilepsy surgery in children with tuberous sclerosis complex: presurgical evaluation and outcome," Epilepsia, vol. 41, no. 9, pp. 1206-1213, 2000.

[28] K. Kagawa, D. C. Chugani, E. Asano et al., "Epilepsy surgery outcome in children with tuberous sclerosis complex evaluated with $\alpha$-[11C]methyl-L-tryptophan positron emission tomography (PET)," Journal of Child Neurology, vol. 20, no. 5, pp. 429-438, 2005.

[29] D. K. Lachhwani, E. Pestana, A. Gupta, P. Kotagal, W. Bingaman, and E. Wyllie, "Identification of candidates for epilepsy surgery in patients with tuberous sclerosis," Neurology, vol. 64, no. 9, pp. 1651-1654, 2005.

[30] T. Kamimura, J. Tohyama, M. Oishi et al., "Magnetoencephalography in patients with tuberous sclerosis and localization-related epilepsy," Epilepsia, vol. 47, no. 6, pp. 991-997, 2006.

[31] H. L. Weiner, C. Carlson, E. B. Ridgway et al., "Epilepsy surgery in young children with tuberous sclerosis: results of a novel approach," Pediatrics, vol. 117, no. 5, pp. 1494-1502, 2006.

[32] F. E. Jansen, A. C. van Huffelen, P. C. van Rijen et al., "Epilepsy surgery in tuberous sclerosis: the Dutch experience," Seizure, vol. 16 , no. 5, pp. 445-453, 2007.

[33] F. Teutonico, R. Mai, O. Devinsky et al., "Epilepsy surgery in tuberous sclerosis complex: early predictive elements and outcome," Child's Nervous System, vol. 24, no. 12, pp. 14371445, 2008.

[34] P. Major, S. Rakowski, M. V. Simon et al., "Are cortical tubers epileptogenic? Evidence from electrocorticography," Epilepsia, vol. 50, no. 1, pp. 147-154, 2009.

[35] J. L. Wen, J. X. Liao, L. Chen et al., "Follow-up of tuberous sclerosis complex complicated by epilepsy in children," Chinese Journal of Contemporary Pediatrics, vol. 11, no. 12, pp. 996-998, 2009.

[36] A. van der Heide, A. C. van Huffelen, W. P. J. Spetgens, C. H. Ferrier, O. van Nieuwenhuizen, and F. E. Jansen, "Identification of the epileptogenic zone in patients with tuberous sclerosis: concordance of interictal and ictal epileptiform activity," Clinical Neurophysiology, vol. 121, no. 6, pp. 842-847, 2010.

[37] S. Liang, A. Li, M. Zhao et al., "Epilepsy surgery in tuberous sclerosis complex: emphasis on surgical candidate and neuropsychology," Epilepsia, vol. 51, no. 11, pp. 2316-2321, 2010. 


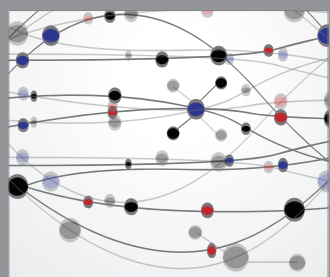

The Scientific World Journal
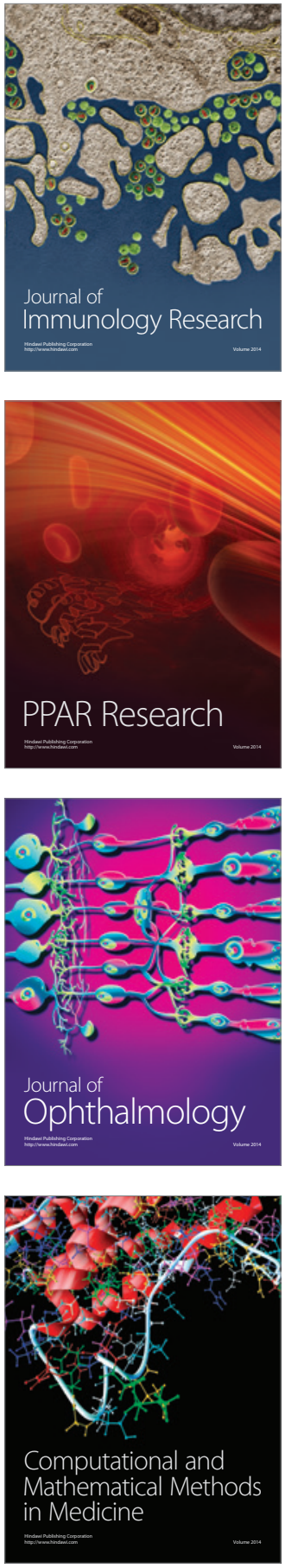

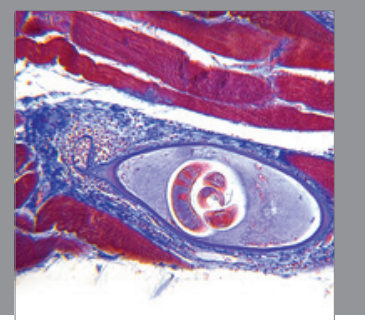

Gastroenterology

Research and Practice
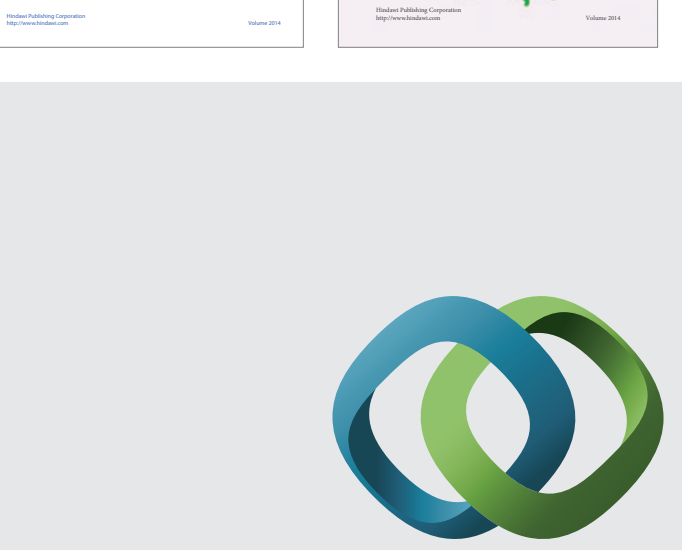

\section{Hindawi}

Submit your manuscripts at

http://www.hindawi.com
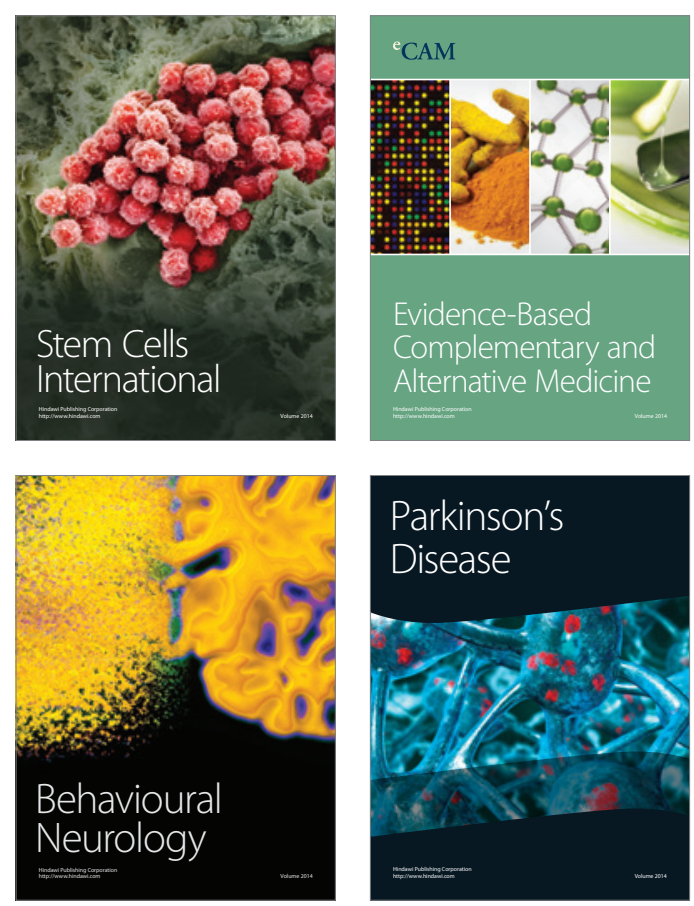

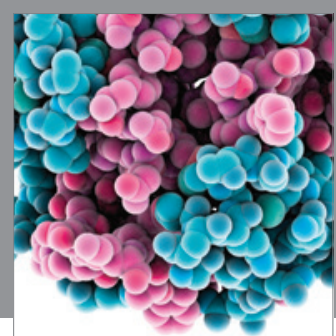

Journal of
Diabetes Research

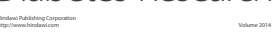

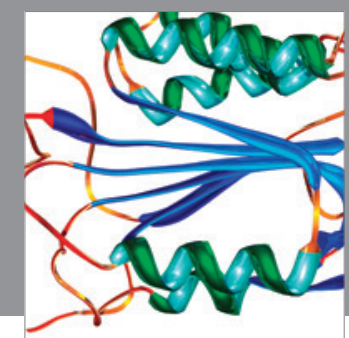

Disease Markers
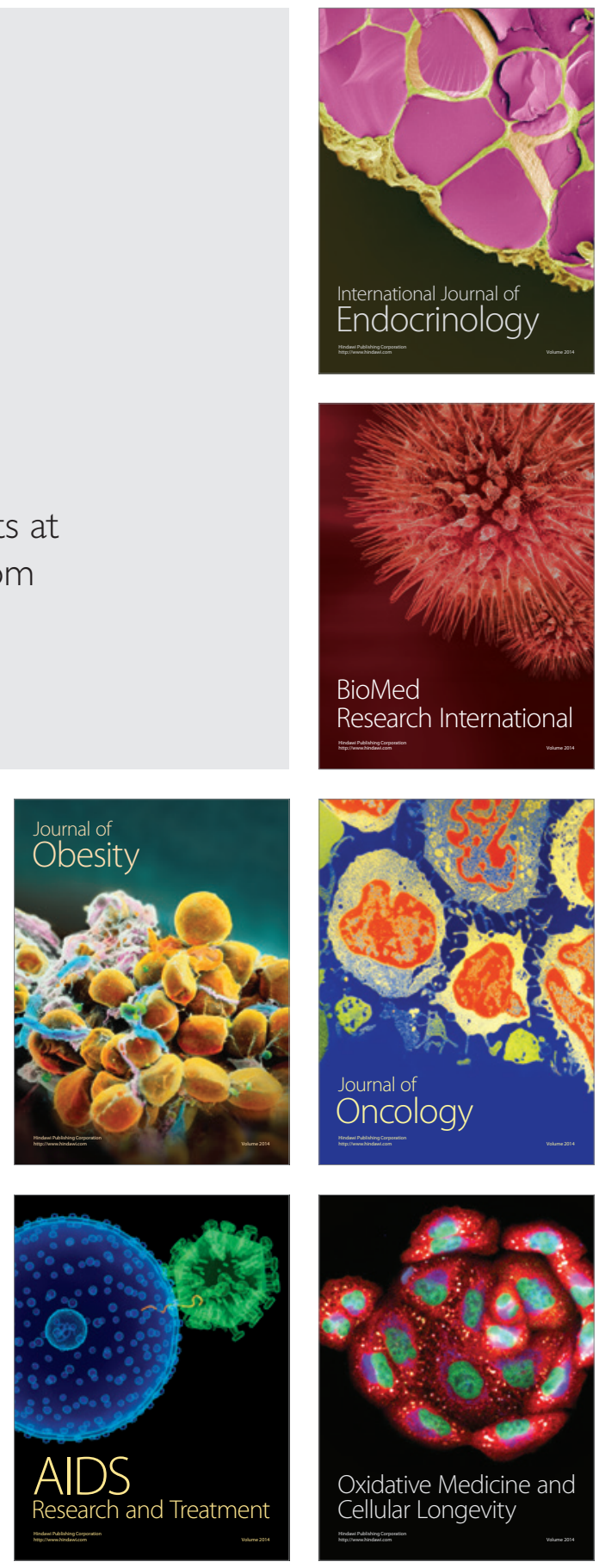